I N S T I T U T O

$\mathrm{DE}$

M E D I C I N A

T R O P I C A L

$\mathrm{DE}$

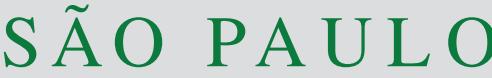

JOURNAL OF THE SÃO PAULO INSTITUTE OF TROPICAL MEDICINE

'Universidade de São Paulo, Faculdade de Medicina, Departamento de Moléstias Infecciosas e Parasitárias, São Paulo, São Paulo, Brazil

2Universidade de São Paulo, Instituto de Medicina Tropical de São Paulo, Laboratório de Virologia, São Paulo, São Paulo, Brazil

${ }^{3}$ Secretaria da Saúde de São Paulo, Fundação Pró-Sangue Hemocentro de São Paulo, São Paulo, São Paulo, Brazil

${ }^{4}$ Secretaria da Saúde de São Paulo, Superintendência de Controle de Endemias, Núcleo de Estudos em Malária, São Paulo, São Paulo, Brazil

${ }^{5}$ Universidade de São Paulo, Instituto de Medicina Tropical de São Paulo, São Paulo, São Paulo, Brazil

Correspondence to: Mariana Aschar Universidade de São Paulo, Faculdade de Medicina, Departamento de Moléstias Infecciosas e Parasitárias, Av. Dr. Enéas de Carvalho Aguiar, 470, Cerqueira César, CEP 05403-000, São Paulo, SP, Brazil Tel: + $55113061-7013$

E-mail: mariana_aschar@usp.br

Received: 15 October 2020

Accepted: 30 November 2020

\section{The hidden Plasmodium malariae in blood donors: a risk coming from areas of low transmission of malaria}

\author{
Mariana Aschar ${ }^{(1)}$, José Eduardo Levi ${ }^{(1,2}$, Maria L. R. N. Farinas ${ }^{(1)}$, Sandra \\ C. Montebello ${ }^{3}$, Alfredo Mendrone-Junior ${ }^{\left({ }^{3} 3\right.}$, Silvia Maria Di Santi ${ }^{(1,4,5}$
}

\section{ABSTRACT}

Malaria is an infectious vector-borne disease with other important routes of transmission, such as blood transfusion and organ/tissue transplantation, due to asymptomatic reservoirs of Plasmodium presenting with low parasitemia. Reports of transfusion-transmitted malaria have shown that in immunosuppressed recipients, infections can be fatal if they are not diagnosed and timely treated. All Plasmodium species can survive on blood components at temperatures from 2 to $6{ }^{\circ} \mathrm{C}$ for some days or even weeks. This report describes two candidates for blood donation harboring Plasmodium, infected in an area considered non-endemic. Blood samples were collected from donors who attended a blood bank in Sao Paulo and tested by microscopy, qPCR for Plasmodium genus-specific amplification, targeting the parasite $18 \mathrm{~S}$ ribosomal subunit gene and a multiplex qPCR based on mtDNA of the five species. Under microscopy, only structures resembling Plasmodium were observed. The qPCR whose standard curve tested parasites varying from 2 to 0.1 parasites/ $\mu \mathrm{L}$, showed the presence of Plasmodium DNA in the two blood donors, as did the multiplex qPCR that revealed the presence of P. malariae. The prevalence of positive donors varies according to the level of transmission, ranging from 0.7 to $55 \%$ in endemic areas. In non-endemic regions, prevalences are lower, however, transfusion malaria can evolve to severe cases, due to the lack of suspicion of this transmission route. Asymptomatic donors from low transmission regions pose a risk to blood banks, with particular emphasis on those located in areas with malaria elimination goals.

KEYWORDS: Malaria. Molecular diagnosis. Transfusion-transmitted malaria. Plasmodium malariae. Blood banks.

\section{INTRODUCTION}

Malaria is an infectious vector-borne disease caused by five Plasmodium species. There are other important routes of transmission, such as by blood transfusion and organ or tissue transplantation, due to asymptomatic reservoirs of Plasmodium presenting with low parasitemia ${ }^{1}$. The Amazon region accounts for $99.6 \%$ of malaria cases in Brazil. The extra-Amazon region is considered non-endemic, since most cases are imported from endemic areas of Brazil or other countries ${ }^{2}$. In addition, sporadic autochthonous cases of $P$. vivax and $P$. malariae are reported in the Atlantic Forest biome, presenting with extremely low parasitemia, mild or absent clinical symptoms and normal hemoglobin levels ${ }^{3}$. Sao Paulo State, located in the extra-Amazon region, reported 113 cases of malaria in 2019, with 14 classified as autochthonous, including cases transmitted in the cities of Paraibuna and Juquitiba, located in the Atlantic Forest Biome ${ }^{4}$. Asymptomatic cases have an impact not 
only on the vectorial transmission, but also on transfusiontransmitted malaria (TTM). Reports of TTM have shown that in immunosuppressed recipients, infections can be fatal if they are not diagnosed and timely treated ${ }^{5}$. The World Health Organization (WHO) preconizes that all blood donations should be screened for malaria, but the lack of consensus on which test to use makes this recommendation difficult to follow. According to the Brazilian guidelines ${ }^{6}$, donor screening criteria differ in endemic and non-endemic areas, as shown in Figure 1. However, criteria adopted for clinical and epidemiological screening are clearly insufficient to detect asymptomatic cases presenting with low parasitemia. For these invisible reservoirs, ultrasensitive diagnostic methodologies are required ${ }^{2}$. The prevalence of asymptomatic infections varies according to the level of malaria transmission. In endemic areas with prevalences by microscopy higher than $70 \%$, asymptomatic infections are about $20 \%$. In areas with microscopy prevalences less than $10 \%$, submicroscopic infections can reach $80 \%$ of the population ${ }^{7}$. In blood transfusion settings, parasites are generally transmitted by infected red blood cells, however, platelets and leukocytes can also transmit malaria through contamination with infected residual erythrocytes. All Plasmodium species can survive in blood components at temperatures from 2 to $6{ }^{\circ} \mathrm{C}$ for some days or even weeks ${ }^{2}$. This report describes two positive candidates for blood donation, diagnosed in a study aiming to detect asymptomatic individuals harboring Plasmodium in blood banks of endemic and non-endemic regions.

\section{MATERIAL AND METHODS}

Blood samples were collected from 292 donors who attended the Pro-Sangue Blood Centre in Sao Paulo, after their informed consent. The first case is a 30-year-old man who traveled to Cuiaba city, Mato Grosso State, Brazil, considered an endemic area for malaria, eight months before the blood donation. According to the Brazilian guidelines ${ }^{6}$, he was tested for the presence of Plasmodium through the thick blood smear (TBS) technique, the reference test in Brazil. The second case is a 27 -year-old woman who travels frequently to Juquitiba, Sao Paulo State, Brazil, a city located in the Atlantic Forest biome, considered a non-endemic area. TBS were performed by two experienced microscopists. For the molecular protocols, DNA was extracted with the QIAamp ${ }^{\circledR}$ DNA Blood Mini Kit (Qiagen, Hilden, Germany). Real time PCR (qPCR) for genus-specific amplification was performed, targeting the 18S rRNA gene of Plasmodium, according to a previous description ${ }^{2}$. Samples were assayed in duplicate in the 7500 Real Time PCR System (Applied Biosystems $^{\mathrm{TM}}$, Thermo Fisher Scientific, Waltham, USA).

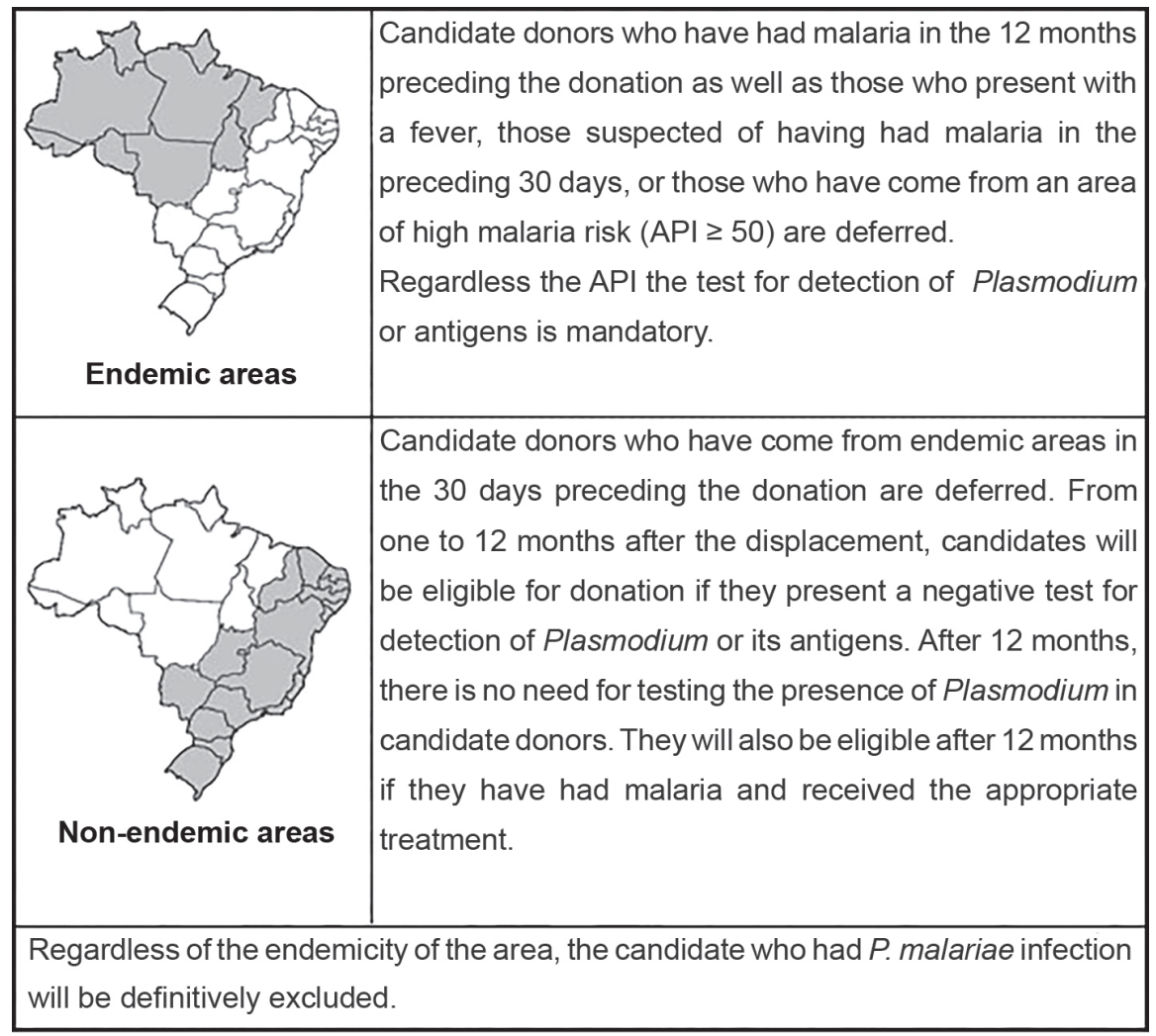

Figure 1 - Brazilian guidelines criteria for screening of blood donation candidates in endemic and non-endemic areas ${ }^{6}$. 
For the differentiation of species, the multiplex Screen \& Type (mqPCR) based on the amplification of mtDNA of $P$. falciparum, $P$. vivax, P. malariae, $P$. ovale and $P$. knowlesi (Altona Diagnostics, Hamburg, Germany) was used.

\section{RESULTS}

In the TBS of the first case, only one structure resembling Plasmodium was observed, but qPCR confirmed the presence of Plasmodium DNA, with a cycle threshold value of 33.1, equivalent to 2 parasites/ $\mu \mathrm{L}$ based on the standard curve. The mqPCR revealed the presence of P. malariae. The second case did not mention travels to endemic areas. TBS was inconclusive after two expert readings, but qPCR was positive for Plasmodium, with a cycle threshold value of 35.3 , equivalent to 0.1 parasite $/ \mu \mathrm{L}$. Plasmodium species was determined by mqPCR identifying P. malariae. These two candidates were asked by the blood bank to attend the reference center for malaria (SUCEN/ HCFMUSP) for treatment according to the Brazilian Ministry of Health guidelines ${ }^{8}$.

\section{DISCUSSION}

TTM represents the most common parasitic infectious disease transmitted by blood. All five human Plasmodium species have already been implicated in TTM events ${ }^{9}$. Reports on prevalence of positive donors showed variable results. Screening candidates using malaria pigment detection by an automated hematology analyzer showed a prevalence of $0.7 \%$ in Kenya. In Nigeria, the prevalence detected by microscopy was $55 \%{ }^{10}$. In nonendemic regions, the prevalence of TTM is lower than in endemic areas, with less than one positive case in one million donations. However, TTM can progress to severe or even fatal malaria due to lack of recipient immunity ${ }^{5}$. According to the Alliance of Blood Operators, blood banks decision-making can vary in different countries. In France, candidates that were born or lived in endemic areas in the first 5 years of life are tested. In England, donors who returned more than 12 months before the donation are accepted. Donors with a history of malaria are not accepted for three years in the United States of America and permanently in Canada ${ }^{11}$.

Serological assays have been used in European countries to screen blood donor candidates ${ }^{12}$. However, the lack of accurate and sensitive protocols, suitable for processing a large number of samples, poses a challenge for the application of these protocols in the routine of blood banks. Mangano et al. ${ }^{13}$ evaluated five commercial ELISA kits for the detection of anti-Plasmodium antibodies and the results showed a sensitivity below $65 \%$ using the indirect fluorescence assay (IFA) as a reference, leading to a high proportion of false negative results. A similar finding was reported in a case of TTM in Georgia, USA, as the donor also had a negative ELISA result ${ }^{14}$. In addition, in a survey aimed at detecting asymptomatic candidates from a low endemic area in Brazil, an individual with positive PCR for P. malariae was negative in the IFA-Pm² ${ }^{2}$ Similar findings were reported by Assennato et al. ${ }^{15}$, who detected P. malariae only by PCR among seronegative donors. However, all decision-making strategies consider the information of donors referring displacements to endemic regions, where symptomatic cases are more prevalent. To mitigate the risk of TTM, it is crucial to evaluate candidates living or referring displacements to areas of low transmission or seeking to eliminate malaria, where asymptomatic infections are reported ${ }^{2}$.

TTM can lead to severe cases more frequently than malaria transmitted by the vector. In this case, parasites undergo the pre-erythrocytic stage during which innate immunity is activated to protect the host; however, parasites transmitted through blood transfusion are released directed into the bloodstream, without performing the hepatic cycle $^{12}$. In immunocompromised recipients, TTM can even lead to fatal cases ${ }^{5,9}$.

During the epidemiological investigation of the first case, the candidate reported a displacement only to the urban area of Cuiaba. However, he has also reported frequent travels to Paraibuna, a municipality located in the Atlantic Forest region in Sao Paulo State, where he usually spends the weekends. Based on the epidemiological characteristics of both locations, the place of infection was defined as Paraibuna, reinforcing the importance of considering asymptomatic hosts infected in areas of low transmission.

In the second case, Plasmodium DNA was detected because the candidate was included in a study to validate sensitive molecular protocols for screening in the context of blood banks and organ transplantation. As tests for the detection of Plasmodium are mandatory only for candidates traveling to endemic regions ${ }^{6}$, after negative results by other tests recommended for screening, the blood unit was transfused into a recipient admitted with traumatic brain injury and ischemic stroke. The recipient died six days after the transfusion, making the possibility of TTM as the cause of death unlikely, as the average incubation period for P. malariae transmitted by blood is 57.2 days $^{12}$ and no signs of malaria infection were reported in the medical record. Even with reports of shorter incubation periods, a fatal outcome in such a few days is unlikely, as P. malariae has a slow growth rate due to its preference for senescent erythrocytes ${ }^{2}$. 
Despite control and prevention measures, autochthonous cases have been identified in several non-endemic regions. Some studies have reported autochthonous cases of malaria in the Atlantic coast of Sao Paulo State, and in more inland areas covered by Atlantic Forest, where vectors of the subgenus Kerteszia are found in large densities, transmitting parasites to humans. The finding of primates infected with Plasmodium reveals that transmission in this region has a zoonotic profile ${ }^{3}$.

The candidates reported in this study had no symptoms at the time of blood screening, what is usual in infections by $P$. malariae. According to some authors, this species has a well-adapted relationship with hosts, who can develop a chronic infection for decades, without symptoms. These infections are obviously not reported to the surveillance systems, and most symptomatic cases detected in this region are usually associated with infections by $P$. vivax ${ }^{3}$.

Asymptomatic donors usually present with low parasitemia, that is not detected under microscopy. Therefore, it is essential to consider that even parasitemia as low as 1 parasite/ $\mathrm{LL}$ will corresponds to 400,000 parasites per unit of whole blood ${ }^{2}$. To face this challenge, the screening of candidates must consider the use of molecular tools to prevent TTM, including those coming from low transmission areas. The choice of the molecular platform for donor screening should use sensitive and easy-to-do assays. In this study, a sensitive genus-specific qPCR was used for donor screening and positive samples were assayed by mqPCR based on the amplification of mtDNA from all species of Plasmodium. Other authors have reported the usefulness of mtDNA qPCR for the detection of $P$. vivax $P$. falciparum and $P$. malariae, carried out in three different reactions. In a study conducted in the Brazilian Amazon region, 10 of 2,224 donor candidates were positive for $P$. vivax $(0.45 \%)^{16}$. Our results detected two cases of P. malariae in 292 donor candidates tested $(0.7 \%)$. Although conventional PCR targeting mtDNA ${ }^{17}$ and $18 \mathrm{SRNA}^{18}$ is available, these protocols are time-consuming and do not apply to blood banks.

This report shows the need for more specific guidelines, including ultrasensitive testing for donors coming from low transmission areas, that are not considered endemic for malaria, where asymptomatic cases can pose a risk for blood banks ${ }^{2,19}$. Similar findings may be associated with areas seeking to eliminate malaria. Despite the large number of blood donor candidates from endemic areas tested in our service, we had never found positive results among them. These candidates for blood donation are mainly individuals who have traveled to endemic areas, without any immunity to the disease. If they had already been infected, they would probably have the classic symptoms of malaria within
30 days of return. On the other hand, candidates from areas with low transmission may present a semi-immune profile due to their frequent exposure to the parasite, presenting with mildly symptomatic infections or even asymptomatic ones, representing a greater risk for blood donation ${ }^{2}$. Low parasitemia is associated with residual immunity, acquired after repeated exposure to Plasmodium. In addition, in some hot spots located in areas of low transmission, individuals can be exposed to the parasite, with the development of immunity. In this scenario, submicroscopic parasitemias in low-endemic settings are the consequence of an effective immunity mechanism, as a result of limited exposure to malaria ${ }^{7}$. Confirming this pattern, all TTMs that we detected were caused by asymptomatic donors harboring $P$. malariae, infected in an extremely low transmission area.

\section{ACKNOWLEDGMENTS}

We gratefully appreciate the support of the staff of Núcleo de Estudos em Malária/SUCEN/IMT - FMUSP and Fundação Pró-Sangue Hemocentro de São Paulo. We also acknowledge the support of Laboratório de Investigação Médica/HCFMUSP (LIM 49).

\section{AUTHORS' CONTRIBUTIONS}

MA designed the study, collect samples, carried out and analyzed the molecular assays and wrote the manuscript; JEL designed the study and reviewed the manuscript; MLRNF carried out the molecular assays; SCM organized samples collection and reviewed the manuscript; AMJ provided data from donors and reviewed the manuscript; SMDS designed the study, coordinated and analyzed the molecular assays and wrote the manuscript. All authors read and approved the final manuscript.

\section{FUNDING}

This work was supported by the Coordenação de Aperfeiçoamento de Pessoal de Nível Superior - Brasil (CAPES), grant $\mathrm{N}^{\circ}$ 88882.376831/2019-01 to MA; Núcleo de Estudos em Malaria/SUCEN and Fundação de Amparo à Pesquisa do Estado de São Paulo (FAPESP), grant $\mathrm{N}^{\circ}$ 2014/50093-8.

\section{REFERENCES}

1. Pierrotti LC, Levi ME, Di Santi SM, Segurado AC, Petersen E. Malaria Disease recommendations for solid organ transplant recipients and donors. Transplantation. 2018;102 Suppl 2:S1626. 
2. Lima GF, Arroyo Sanchez MC, Levi JE, Fujimori M, Da Cruz Caramelo L, Sanchez AR, et al. Asymptomatic infections in blood donors harbouring Plasmodium: an invisible risk detected by molecular and serological tools. Blood Transfus. 2018;16:17-25.

3. Hristov AD, Arroyo Sanchez MC, Ferreira JJ, Lima GF, Inoue $\mathrm{J}$, Costa-Nascimento MJ, et al. Malaria in pregnant women living in areas of low transmission on the southeast Brazilian Coast: molecular diagnosis and humoural immunity profile. Mem Inst Oswaldo Cruz. 2014;109:1014-20.

4. Brasil. Ministério da Saúde do Brasil. Secretaria de Vigilância em Saúde. Boletim região Extra-Amazônica: 19/10/2020. [cited 2020 Nov 30]. Available from: https://public.tableau. com/profile/mal.ria.brasil\#!/vizhome/BoletimregiaoExtraAmazonica_19_10_2020/SrieHist

5. Kirchgatter K, Nogueira SL, Padilha A, Curado I, Boulos M, Di Santi SM. Lethal malaria caused by Plasmodium malariae in an asplenic patient in Brazil. BMJ. 2005;331:576.

6. Brasil. Ministério da Saúde do Brasil. Gabinete do Ministro. Portaria $\mathrm{n}^{\circ} 158$, de 4 de fevereiro de 2016. Redefine o regulamento técnico de procedimentos hemoterápicos. Diário Oficial da União, Brasília, 5 fev. 2016. Seção 1:37. [cited 2020 Nov 30] Available from: https://bvsms.saude.gov.br/bvs/ saudelegis/gm/2016/prt0158_04_02_2016.html

7. Bousema T, Okell L, Felger I, Drakeley C. Asymptomatic malaria infections: detectability, transmissibility and public health relevance. Nat Rev Microbiol. 2014;12:833-40.

8. Brasil. Ministério da Saúde. Secretaria de Vigilância em Saúde. Guia de tratamento da malária no Brasil. Brasília: Ministério da Saúde; 2010. [cited 2020 Nov 30]. Available from: https:// bvsms.saude.gov.br/bvs/publicacoes/guia_pratico_malaria.pdf

9. Schindler T, Robaina T, Sax J, Bieri JR, Mpina M, Gondwe L, et al. Molecular monitoring of the diversity of human pathogenic malaria species in blood donations on Bioko Island, Equatorial Guinea. Malar J. 2019;18:9.

10. Owusu-Ofori AK, Parry C, Bates I. Transfusion-transmitted malaria in countries where malaria is endemic: a review of the literature from sub-Saharan Africa. Clin Infect Dis. 2010;51:1192-8.

11. O'Brien SF, Ward S, Gallian P, Fabra C, Pillonel J, Kitchen $\mathrm{AD}$, et al. Malaria blood safety policy in five non-endemic countries: a retrospective comparison through the lens of the $\mathrm{ABO}$ risk-based decision-making framework. Blood Transfus. 2019;17:94-102.

12. Verra F, Angheben A, Martello E, Giorli G, Perandin F, Bisoffi Z. A systematic review of transfusion-transmitted malaria in non-endemic areas. Malar J. 2018;17:36.

13. Mangano VD, Perandin F, Tiberti N, Guerriero M, Migliaccio F, Prato M, et al. Risk of transfusion-transmitted malaria: evaluation of commercial ELISA kits for the detection of
anti-Plasmodium antibodies in candidate blood donors. Malar J. 2019;18:17.

14. Holtzclaw A, Mrsic Z, Managbanag J, Calvano T, Colombo C. Transfusion transmitted malaria not preventable by current blood donor screening guidelines: a case report. Transfusion. 2016;56:2221-4.

15. Assennato SM, Berzuini A, Foglieni B, Spreafico M, Allain JP, Prati D. Plasmodium genome in blood donors at risk for malaria after several years of residence in Italy. Transfusion. 2014;54:2419-24.

16. Batista-Dos-Santos SA, Freitas DR, Raiol M, Cabral GF, Feio AC, Póvoa MM, et al. Strategy to improve malaria surveillance system preventing transfusion-transmitted malaria in blood banks using molecular diagnostic. Malar J. 2018;17:344.

17. Cunha MG, Medina TS, Oliveira SG, Marinho AN, Póvoa MM, Ribeiro-dos-Santos AK. Development of a Polymerase Chain Reaction (PCR) method based on amplification of mitochondrial DNA to detect Plasmodium falciparum and Plasmodium vivax. Acta Trop. 2009;111:35-8.

18. Snounou G, Viriyakosol S, Zhu XP, Jarra W, Pinheiro L, do Rosario VE, et al. High sensitivity of detection of human malaria parasites by the use of nested polymerase chain reaction. Mol Biochem Parasitol. 1993;61:315-20.

19. Dos Santos EH, Yamamoto L, Domingues W, Di Santi SM, Kanunfre KA, Okay TS. A new Real Time PCR with species-specific primers from Plasmodium malariae/ P. brasilianum mitochondrial cytochrome $b$ gene. Parasitol Int. 2020;76:102069.

\section{ERRATUM}

Page 1, where it reads:

Silvia Maria Di Santi

Should be read:

Silvia Maria DI SANTI

http://dx.doi.org/10.1590/s1678-9946202062100err 